\title{
Elevated methylation of the vault RNA2-1 promoter in maternal blood is associated with preterm birth
}

\author{
Young-Ah You', Eun Jin Kwon ${ }^{2}$, Han-Sung Hwang ${ }^{3}$, Suk-Joo Choi ${ }^{4}$, Sae Kyung Choi ${ }^{5}$ and Young Ju Kim ${ }^{1,2,6^{*}}$ (D)
}

\begin{abstract}
Background: Preterm birth, defined as parturition before 37 completed weeks of gestation, is associated with an increased risk of neonatal complications and death, as well as poor health and disease later in life. Epigenetics could contribute to the mechanism underlying preterm birth.

Results: Genome-wide DNA methylation analysis of whole blood cells from 10 women ( 5 term and 5 preterm deliveries) was performed using an Illumina Infinium HumanMethylation450 BeadChips array. We identified 1,581 differentially methylated CpG sites in promoter regions between term and preterm birth. Although the differences were not significant after correcting for multiple tests, seven CpGs on the genomically imprinted vault RNA2-1 (VTRNA2-1; also known as non-coding RNA, nc886 or miR-886) showed the largest differences (range: 26-39\%). Pyrosequencing verification was performed with blood samples from pregnant women recruited additionally (39 term and 43 preterm deliveries). In total, 28 (34.1\%) samples showed hypomethylation of the VTRNA2-1 promoter ( $<13 \%$ methylation), while 54 (65.9\%) samples showed elevated methylation levels between 30 and $60 \%$. Elevated methylation of VTRNA2-1 promoter was associated with an increased risk of preterm birth after adjusting for maternal age, season of delivery, parity and white blood cell count. The mRNA expression of VTRNA2-1 was 0.51fold lower in women with preterm deliveries $(n=20)$ compared with women with term deliveries $(n=20)$.

Conclusions: VTRNA2-1 is a noncoding transcript to environmentally responsive epialleles. Our results suggest that elevated methylation of the VTRNA2-1 promoter may result in increased risk of PTB caused by the pro-inflammatory cytokines. Further studies are needed to confirm the association of VTRNA2-1 methylation with preterm birth in a large population, and to elucidate the underlying mechanism.
\end{abstract}

Keywords: DNA methylation, Maternal blood, VTRNA2-1, Preterm birth, miR-886

\footnotetext{
*Correspondence: kkyj@ewha.ac.kr

${ }^{1}$ Department of Obstetrics and Gynecology, Ewha Medical Research Institute,

Ewha Womans University Medical School, 07985 Seoul, Korea

${ }^{2}$ Graduate Program in System Health Science and Engineering, Ewha

Womans University, 03760 Seoul, Korea

Full list of author information is available at the end of the article
}

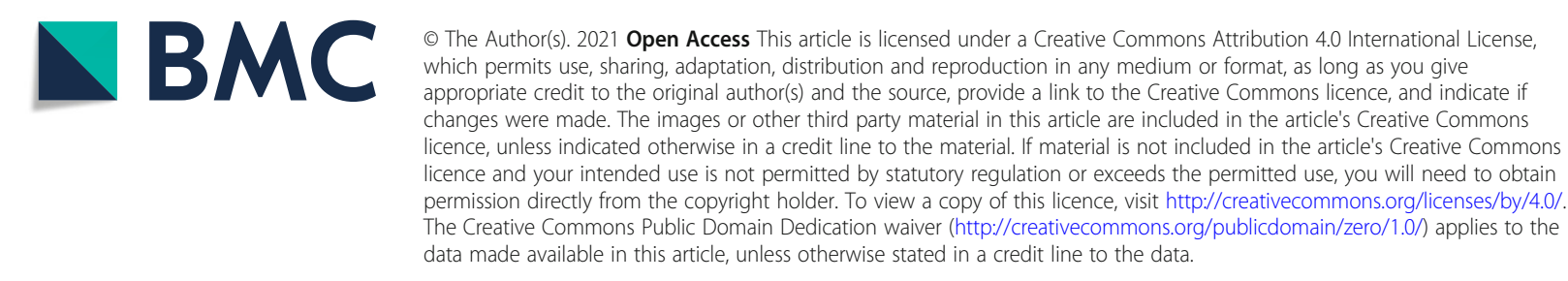




\section{Background}

Preterm birth (PTB) is defined as parturition before 37 weeks of gestation and approximately 15 million babies are born prematurely each year [1]. In Korea, the rate of PTB has continuously increased, from $4.8 \%$ to 2008 to $7.8 \%$ in 2018 [2, 3]. To date, clinicians and researchers have made great efforts to improve the identification of women at risk for PTB before its occurrence, as well as to develop therapeutics for its prevention. However, the early identification of PTB and therapy to mitigate its risk remain controversial $[4,5]$.

PTB is associated with an increased risk of complications and neonatal death, as well as poor health and disease later in life [6-8]. Spontaneous PTB has a range of contributing risk factors, including infection, undernutrition, stress, and substance use [9], which are linked by two common pathways [10]. First, inflammatory and neuroendocrine pathways are activated in response to stress or stressrelated behaviours, such as smoking [11], causing the upregulation of inflammatory cytokine production [11]. Second, cytokine-prostaglandin cascades are activated in response to infection [10]. Due to the involvement of inflammatory mechanisms in both pathways, previous studies investigating potential biomarkers for PTB have focused on inflammatory mediators $[12,13]$.

Genome-wide DNA methylation analysis may provide information on the mechanism underlying PTB and represents a new approach for biomarker discovery [14-16]. DNA methylation in the blood may change according to conditions such as inflammation, and multiple studies have shown that differential DNA methylation is related to smoking status, as well as to obesity and various other diseases [17]. Moreover, the ability to assess the epigenome has resulted in the identification of epigenetic signatures of the intrauterine environment, which are affected by smoking, stress, nutrition, body mass index (BMI), and medication use over several years [18-22]. Many studies have suggested that differentially methylated genes are involved in the genetic and environmental contributions to PTB and chronic disease risk. DNA methylation changes in the amnion or foetal tissue were determined to be partially involved in the physiological process of PTB and in foetal development [23, 24]. Thus, the evaluation of genes containing these differentially methylated sites may be useful to identify biological pathways involved in PTB, thereby facilitating the identification of clinically informative biomarkers for the prediction of PTB. Accordingly, we investigated the association between blood DNA methylation and preterm birth among Korean women. We identified the seven CpG sites with a difference in VTRNA2-1 promoter methylation levels between preterm and term samples.

VTRNA2-1 (VTRNA2-1, also called nc886 or miR-886) is a 108-nucleotide noncoding transcript that is epigenetically controlled via $18 \mathrm{CpG}$ sites of its promoter, and can exert either tumor suppression or oncogenic functions depending on cell types of cancers [25]. Intriguing aspects of the epigenetic regulation of this locus, include its dependence on the parental origin of the allele, and its sensitivity to the periconceptional environment $[26,27]$. Here, we report that the elevated methylation of VTRNA2-1 promoter, validated by pyrosequencing after an epigenome-wide analysis, is associated with preterm birth.

\section{Results \\ Methylation array in the peripheral blood of women delivering at term and preterm}

A genome-wide analysis of DNA methylation was performed to search for candidate differentially methylated genes in maternal blood at the time of delivery. A total of 87,507 CpG sites were tested on the CpG island promoters (except SNP-affected CpG sites) and the results showed that 1,581 sites had significantly different levels of methylation $(p<0.05)$ between term and preterm groups (Table S1). Although we found no differential methylation (DM) of these CpGs after correcting for multiple testing, seven CpG sites in the VTRNA2-1 promoter showed the largest differences (26-39\%) between PTB and term samples $(p<0.05)$ (Table 1$)$. In addition, these seven $\mathrm{CpG}$ sites showed elevated methylation (range: $25.0-55.1 \%$ ) in all 5 samples of term deliveries and 1 sample of preterm delivery, and hypomethylation (range: $2.6-14.1 \%$ ) in 4 samples of preterm deliveries (Figure S1).

\section{Validation of VTRNA2-1 methylation level by pyrosequencing}

Subsequently, we performed pyrosequencing to investigate the association between VTRNA2-1 methylation (cg04481923) and PTB in maternal blood samples (term, $n=39$; preterm, $n=43$ ). The primer sets for the VTRN A2-1 CpG sites were designed using PSQ Assay Design software (Biotage AB, Uppsala, Sweden). We performed pyrosequencing on cg04481923, where the primer set was designed with the highest score among seven CpGs. Table 2 shows the clinical characteristics of the 82 pregnant women. Maternal age ranged from 27 to 39 years in women with term deliveries, and from 22 to 43 years in women with PTB. Among the preterm delivery mothers, 21 were mothers with preterm premature rupture of membrane (pPROM), and 22 were mothers with preterm labor (PTL). The mean gestational age and BMI of women with term births were 39 weeks, 4 days and 26.0, respectively, compared to 29 weeks, 1 day and 23.8, respectively, in women with $\mathrm{PTB}$. The incidence of Csection did not differ significantly between the term and preterm groups according to the chi-square test $(p>$ 
Table 1 The seven CpGs on VTRNA2-1 with the largest differences in methylation between term and preterm samples $(n=10)$

\begin{tabular}{lllllllcc}
\hline Target IDt & CHR & Position & $\begin{array}{l}\text { Functional } \\
\text { location }\end{array}$ & $\begin{array}{l}\text { Mean } \boldsymbol{\beta} \text { value in term } \\
\text { samples }(\boldsymbol{n}=\mathbf{5})\end{array}$ & $\begin{array}{l}\text { Mean } \boldsymbol{\beta} \text { value in preterm } \\
\text { samples }(\boldsymbol{n}=\mathbf{5})\end{array}$ & Difference* $^{*}$ & $\boldsymbol{p}_{\text {-value }}{ }^{\mathbf{a}}$ & $\boldsymbol{q}_{\text {-value }} \mathbf{b}^{\mathbf{b}}$ \\
\hline cg26328633 & 5 & $135,416,394$ & TSS200 & 0.125 & 0.520 & 0.395 & 0.014 & 0.050 \\
cg25340688 & 5 & $135,416,398$ & TSS200 & 0.121 & 0.508 & 0.387 & 0.014 & 0.050 \\
cg06536614 & 5 & $135,416,381$ & TSS200 & 0.129 & 0.492 & 0.363 & 0.020 & 0.050 \\
cg00124993 & 5 & $135,416,412$ & TSS200 & 0.114 & 0.471 & 0.357 & 0.014 & 0.050 \\
cg26896946 & 5 & $135,416,405$ & TSS200 & 0.192 & 0.503 & 0.311 & 0.013 & 0.050 \\
cg04481923 & 5 & $135,416,205$ & 1stExon;3'UTR & 0.122 & 0.389 & 0.267 & 0.015 & 0.050 \\
cg18678645 & 5 & $135,416,331$ & TSS200 & 0.115 & 0.376 & 0.261 & 0.051 & 0.050 \\
\hline
\end{tabular}

Gene ID of VTRNA2-1 is 100,126,299

CHR Chromosome

a Mann-Whitney U-test, $p<0.05$

${ }^{\mathrm{b}}$ The false discovery rate (FDR) was controlled using the Benjamini-Hochberg correction $(q<0.05)$

†Name of the probe

*Difference between mean $\beta$ values of preterm births and term births

0.05). However, white blood cell (WBC) count and monocyte portion in the maternal blood were significantly different between term and preterm delivered women.

Table 3 shows the levels of DNA methylation of three CpG sites on VTRNA2-1 promoter following pyrosequencing; two $\mathrm{CpG}$ sites differed significantly between pregnant women with term births and PTBs $(p<0.05)$. These three CpG sites on VTRNA2-1, identified in all samples, were concordant with hypomethylation (range: $0-13 \%, n=28$ ) or elevated methylation (range: $30-60 \%$, $n=54$ ) (Fig. 1a). Blood samples from women who delivered preterm infants were more likely to exhibit elevated methylation (>30\%) of VTRANA2-1 than women who delivered at term. In addition, the relative expression level of VTRNA2-1 was 0.51 -fold lower $(p<0.05)$ in PTB women $(n=20)$ compared to those with term deliveries $(n=20)$ (Fig. 1b). The methylation levels of the VTRNA2-1 promoter were inversely correlated with levels of its expression $(r=-0.741, p<0.01)$.

To evaluate the clinical relevance of VTRNA2-1 promoter methylation levels, we analysed its association with the demographic and clinical data of pregnant women. The elevated methylation of the VTRNA2-1 promoter was significantly associated with the diagnosis of pPROM, a main cause of PTB (Table 4).

\section{Preterm birth-related DNA methylation changes in maternal blood}

Table 5 shows the association between DNA methylation sites and PTB, as determined by logistic regression analysis. It was divided into two groups based on the methylation level of VTRNA2-1 promoter: a hypomethylated group $(<13 \%, n=28)$ and an elevated methylated group $(30-60 \%, n=54)$. In total, 28 (34.1\%) samples showed elevated methylation of the VTRNA2-1 promoter (<13\% methylation by pyrosequencing), while 54 samples $(65.9 \%)$ showed a methylation level of 30-60\%. Based on these results, the patients were divided into hypomethylation and elevated methylation groups. The elevated methylation of VTRNA2-1 promoter was associated with a significantly increased risk of PTB compared with hypomethylation after adjusting for maternal age, season of delivery, parity and white blood cell count (adjusted OR $=3.358,95 \%$ CI 1.114-10.126). Interestingly, younger women in the VTRNA2-1 hypomethylation group were more likely to have preterm deliveries $(p<0.05$, Table 6). Moreover, women with preterm deliveries in the elevated methylation group had lower BMIs and higher WBC counts and were also more likely to deliver during spring or summer than autumn or winter $(p<0.05)$.

\section{Discussion}

We examined PTB-related DNA methylation changes through genome-wide methylation analysis of maternal blood. Our results showed for the first time that elevated methylation of the VTRNA2-1 promoter in maternal blood is more apparent in pPROM samples than PTL samples, and may increase risk of PTB. In concordance with methylation status, its expression was downregulated in preterm blood and upregulated in term blood samples. Our results suggest that elevated methylation of VTRNA2-1 promoter is susceptible to PTB.

PTB remains the leading cause of childhood morbidity and death. Its aetiology remains unclear; however, significant advances have been made in the identification of biomarkers to predict high-risk pregnancies resulting in PTB. One study using genome-wide methylation analysis reported that maternal methylation loci may serve as a biomarker for PTB, whereas cord blood methylation levels are not associated with PTB [28]. However, other studies have reported that numerous $\mathrm{CpG}$ sites in the cord blood are differentially methylated in relation to 
Table 2 Clinical characteristics of the study groups ( $n=82$ )

\begin{tabular}{|c|c|c|c|}
\hline & Term $(\geq 37, n=39)$ & Preterm $(<37, n=43)$ & $p$-value \\
\hline & Mean \pm SD & Mean \pm SD & \\
\hline Maternal age & $31.7 \pm 2.8$ & $30.9 \pm 4.6$ & 0.418 \\
\hline BMI at delivery & $25.9 \pm 3.3$ & $24.1 \pm 4.2$ & $0.037^{\mathrm{a}}$ \\
\hline Gravidity & & & 0.471 \\
\hline 0 & $16(41.0)$ & $15(34.9)$ & \\
\hline 1 & $23(59.0)$ & $28(65.1)$ & \\
\hline Parity & & & 0.118 \\
\hline Nulliparous & $22(56.4)$ & $22(51.2)$ & \\
\hline Multiparous & $17(43.6)$ & $21(48.8)$ & \\
\hline Delivery season & & & $0.021^{*}$ \\
\hline Spring, $n(\%)$ & $5(12.8)$ & $12(27.9)$ & \\
\hline Summer, $n(\%)$ & $3(7.7)$ & $11(25.6)$ & \\
\hline Autumn, $n(\%)$ & 19 (48.7) & $13(30.2)$ & \\
\hline Winter, n (\%) & $12(30.8)$ & $7(16.3)$ & \\
\hline Mode of delivery & & & 0.599 \\
\hline Vaginal, $n(\%)$ & $24(61.5)$ & $24(55.8)$ & \\
\hline C-section, $n(\%)$ & $15(38.5)$ & $19(44.2)$ & \\
\hline Education $(n=68)$ & & & 0.096 \\
\hline Below high school & $8(20.5)$ & $13(44.8)$ & \\
\hline College or more & $31(79.5)$ & $16(55.2)$ & \\
\hline Gestational age & $39.4 \pm 1.0$ & $29.1 \pm 2.8$ & $<0.001^{\mathrm{a}}$ \\
\hline White blood cell, ( $x 10^{3}$ cells $\left./ \mu \mathrm{L}\right)$ & $10.10 \pm 3.00$ & $12.21 \pm 3.83$ & $0.008^{a}$ \\
\hline Granulocyte, (\%) & $74.65 \pm 7.44$ & $77.81 \pm 7.01$ & 0.066 \\
\hline Lymphocyte, (\%) & $18.01 \pm 5.78$ & $21.55 \pm 34.72$ & 0.548 \\
\hline Monocyte, (\%) & $6.86 \pm 2.01$ & $5.81 \pm 1.76$ & $0.021^{\mathrm{a}}$ \\
\hline Mycoplasma & & & 0.702 \\
\hline Positive, $n(\%)$ & $2(5.4)$ & $3(8.6)$ & \\
\hline Negative, $n(\%)$ & 35 (94.6) & $32(91.4)$ & \\
\hline Ureaplasma $(n=72)$ & & & $<0.001^{*}$ \\
\hline Positive, $n$ (\%) & $0(0.0)$ & $13(37.1)$ & \\
\hline Negative, $n(\%)$ & $37(100.0)$ & $22(62.9)$ & \\
\hline Chorioamnionitis ( $n=67$ ) & & & $<0.001^{*}$ \\
\hline Positive, $n$ (\%) & $0(5.1)$ & $17(56.7)$ & \\
\hline Negative, $n(\%)$ & $37(100)$ & $13(43.3)$ & \\
\hline Birth weight & $3310.5 \pm 394.7$ & $1385.7 \pm 504.1$ & $<0.001^{\mathrm{a}}$ \\
\hline Sex & & & 0.654 \\
\hline Male, $n(\%)$ & $20(51.3)$ & $24(55.8)$ & \\
\hline Female, $n(\%)$ & 19 (48.7) & $19(44.2)$ & \\
\hline Apgar 1 min & $9.5 \pm 0.8$ & $5.9 \pm 2.4$ & $<0.001^{a}$ \\
\hline Apgar 5 min & $9.9 \pm 0.2$ & $7.5 \pm 2.1$ & $<0.001^{\mathrm{a}}$ \\
\hline
\end{tabular}

Data are shown as the mean \pm SD for continuous variables and as $n$ (\%) for categorical data $B M I$ body mass index

${ }^{\text {a }}$ Student's t-test

${ }^{*} X^{2}$ test 
Table 3 Comparison of specific DNA methylation sites between women with term and preterm births after pyrosequencing

\begin{tabular}{lllll}
\hline Variable & $\begin{array}{l}\text { Target ID }+ \text { of differentially } \\
\text { methylated site }\end{array}$ & Term $(\boldsymbol{n}=\mathbf{3 9})$ & Preterm $(\boldsymbol{n}=\mathbf{4 3})$ & $\boldsymbol{p}$-value \\
\hline VTRNA2-1_pos1 & cg04481923 & $25.8 \pm 20.9$ & $33.9 \pm 18.6$ & 0.065 \\
VTRNA2-1_pos2 & & $26.3 \pm 22.2$ & $35.2 \pm 19.5$ & $0.045^{\mathrm{a}}$ \\
VTRNA2-1_pos3 & & $27.4 \pm 22.3$ & $36.4 \pm 19.8$ & $0.026^{\mathrm{a}}$ \\
\hline
\end{tabular}

Data are presented as the mean \pm SD

VTRNA2-1_pos1,_pos2, and_pos3 were analyzed by pyrosequencing as CpG sites of chromosome 5:135,416,205, chromosome 5:135,416,207, and chromosome 5:135,416,214, respectively

VTRNA2-1 vault RNA 2 - 1

${ }^{a}$ Mann-Whitney U-test, $p<0.05$

†Name of the probe

gestational age $[29,30]$. Changes in DNA methylation caused by environmental factors regulate gene transcription and can play a role in a variety of diseases [31]. Studies have suggested that maternal factors, including socioeconomic status [11], pre-pregnancy BMI [32], smoking during pregnancy [33] and nutrition status [34] affect the risk of PTB. DNA methylation partially explains the effects many of these factors [32-34]. In this study, we found associations of maternal BMI, delivery season, and WBC count with PTB; however, these factors did not influence the DNA methylation levels of VTRNA2-1 in maternal blood.

The main pathways of initiation of PTB are fetal and maternal tissue activation by cervical insufficiency, stress, inflammation, and immune dysregulation. These risks result in myometrium contractions and/or rupture of the fetal membranes by the release of prostaglandins and interleukins (ILs) [35, 36]. In this study, elevated methylation of the VTRNA2-1 promoter was analysed in blood samples from 18 women out of 21 pregnant women diagnosis with pPROM, and 17 of 27 blood samples were analysed for elevated methylation in PTL. pPROM is the rupture of the membrane before the onset of labor before 37 weeks of gestation and accounts for one-third of all PTBs [37]. High levels of proinflammatory cytokines such as interleukin (IL)1RA, IL$1 \beta$, IL-6, tumor necrosis factor $\alpha$ (TNF- $\alpha$ ), monocyte chemotactic protein-1 have been reported in the plasma of pregnant women with PPROM [38]. In a previous investigation of ours, cytokine levels in the vaginal fluid were higher among pregnant women who delivered preterm with pPROM and PTL compared to women with term deliveries [4]. We suggest that further studies are need to investigate the link between methylation of VTRNA2-1 promoter and cytokine in maternal blood.

VTRNA2-1 is a major candidate for an environmentally responsive epiallele and its expression is regulated to epigenetic silencing by promoter methylation [39], which could be modulated via either stress, chemotherapy or TGF- $\beta$ cytokine activity $[25,34]$. Levels of VTRN A2-1 methylation are similar among normal mature B cells, T cells and granulocytes [25]. In addition, VTRN A2-1 is reported to have DM sites in various regions and is associated with several diseases [25, 26]. Although few studies have investigated the relationship between VTRNA2-1 and PTB, the elevated methylation is
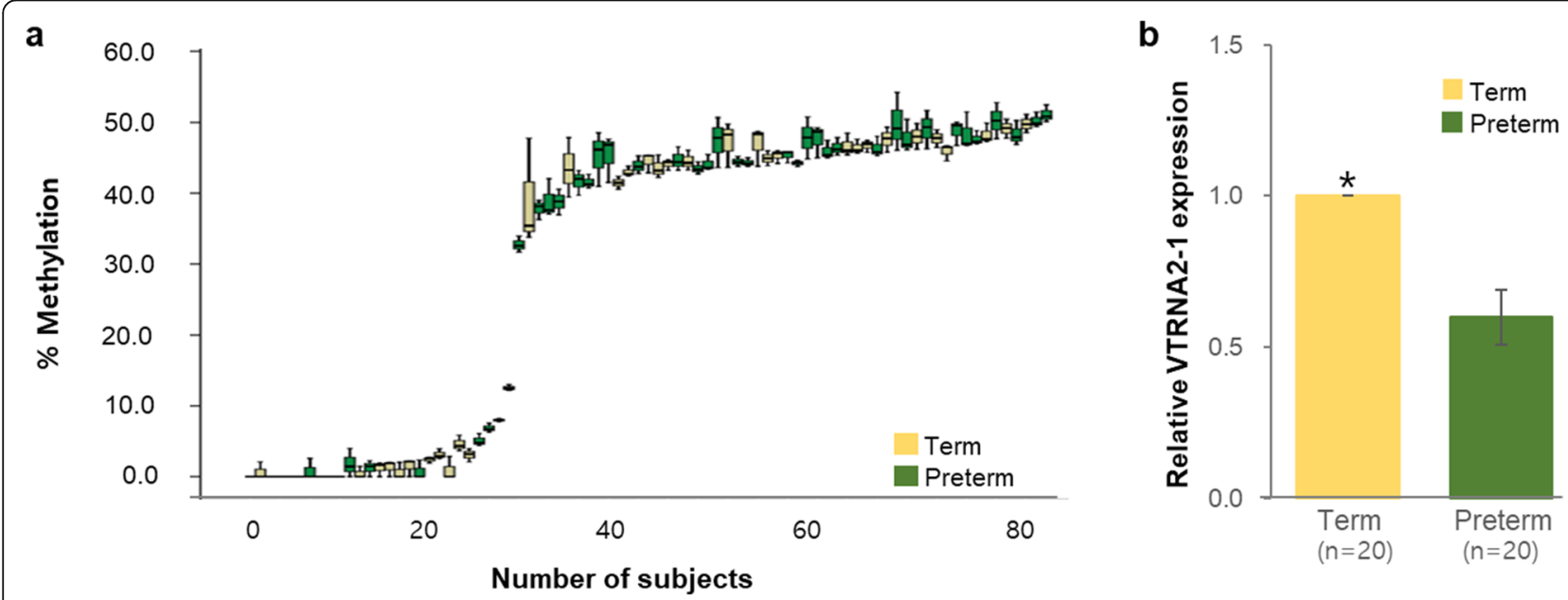

Fig. 1 Methylation differences in VTRNA2-1 between term and preterm samples. a Rank plot of three methylated sites on VTRNA2-1 by bisulphite pyrosequencing. $\mathbf{b}$ Relative expression levels of VTRAN2-1 in maternal blood (Term, $n=20$; Preterm, $n=20$ ) 
Table 4 Correlation analysis of VTRNA2-1 methylation levels and risk factors of preterm birth

\begin{tabular}{llllll}
\hline Variable & Maternal age & Education & Parity & Diagnosis $^{\mathbf{b}}$ & WBC $^{\text {(n) }}$ \\
\hline VTRNA2-1_pos1 & 0.183 & -0.107 & -0.019 & $0.273^{*}$ & 0.105 \\
VTRNA2-1_pos2 & 0.169 & -0.095 & -0.020 & $0.279^{*}$ & 0.104 \\
VTRNA2-1_pos3 & 0.174 & -0.147 & -0.009 & $0.293^{* *}$ & 0.094 \\
Hypo vs. Elevated $^{\text {a }}$ & 0.179 & -0.128 & -0.011 & $0.246^{*}$ & 0.075 \\
\hline
\end{tabular}

The association between the categorical data for VTRNA2-1 methylation and the categorical data for preterm birth risks were analyzed using Fisher's exact test. The association between the others and VTRNA2-1 methylation were analyzed using Pearson correlation analysis

VTRNA2-1_pos1,_pos2, and_pos3 were analyzed by pyrosequencing as CpG sites of chromosome 5:135,416,205, chromosome 5:135,416,207, and chromosome 5:135,416,214, respectively

at divided two groups as hypomethylation (<13\%) and elevated methylation (30-60\%)

${ }^{b}$ Diagnosis were analysed by substituting 1 for PTL, a nominal variable, and 2 for pPROM

${ }^{*} p<0.05$

${ }^{* *} p<0.01$

associated with poor outcomes in patients with acute myeloid leukaemia and small cell lung cancer $[25,26]$. Moreover, inhibition of VTRNA2 increases Bax protein expression and apoptotic cell death in cervical cancer cells [40].

One study reported that the methylation level of VTRNA2-1 was associated with the season of conception and maternal nutrition in rural Gambia [34]. Specifically, the authors reported that women had differing nutrition in the dry and rainy seasons during preconception. However, although our VTRNA2-1 methylation data were associated with season of delivery, it is difficult to explain the relationship between PTB and methylation changes by season. Another studies reported that alterations in DNA methylation result from inflammatory processes, such as circulating levels of C-reactive protein (CRP) or other inflammatory proteins [41, 42]. In this study, level of VTRNA2-1 methylation is correlated with circulating level of CRP in PTB and CRP level is correlated with WBC count (data now shown). Therefore, our study suggests the possible that elevated VTRN A2-1 is susceptible to PTB by inducing infectioninflammation pathways.

Our results showed that expression of VTRNA2-1 was negatively correlated with methylation levels, and that

Table 5 Logistic regression analysis of the association between cg04481923 methylation level in VTRNA2-1 gene and preterm births $(n=82)$

\begin{tabular}{llllll}
\hline Methylation & $\boldsymbol{\beta}$-value & SE & Odds ratio & $\mathbf{9 5 \% ~ C l}$ & $\boldsymbol{p}$-value \\
\hline Age & -0.057 & 0.035 & 0.945 & $0.882-1.012$ & 0.104 \\
Season & -0.582 & 0.238 & 0.559 & $0.350-0.892$ & 0.015 \\
Parity & 0.389 & 0.313 & 1.475 & $0.798-2.727$ & 0.215 \\
VTRNA2-1 $^{\text {a }}$ & 1.185 & 0.574 & 3.269 & $1.061-10.069$ & 0.039 \\
WBC count & 0.211 & 0.075 & 1.235 & $1.066-1.431$ & 0.005 \\
\hline
\end{tabular}

$\beta$-values and SE estimated using the multivariable logistic regression model VTRNA2-1 vault RNA 2 - 1

alt was divided into two groups based on the methylation level of VTRNA2-1 promoter: a hypomethylated group $(<13 \%, n=28)$ and an elevated methylated group $(30-60 \%, n=54)$ elevated methylation of the VTRNA2-1 promoter was associated with PTB. VTRNA2-1 is a putative tumour suppressor and modulator of innate immunity [26]. VTRNA2-1 expression is suppressed in clinical tumour samples compared to normal tissues; more importantly, low expression of VTRNA2-1 is associated with poor survival. In addition, inhibition of VTRNA2-1 leads to activation of the cellular antiviral response pathways involving protein kinase-R (PKR) [43-45]. PKR activation can be induced by various stressful stimuli, such as cytotoxic cytokines, growth factor deprivation and DNA damage [45]. As mentioned above, proinflammatory cytokines are associated with increased risk of PTB. Thus, we propose the possibility that elevated methylation of VTRNA2-1 could cause PTB due to PKR activation in response to increased cytokine.

In our results, the methylation levels of VTRNA2-1 were divided into either a $30-60 \%$ group or $0-13 \%$ group. The pyrosequencing result from our cohort showed hypomethylation at the VTRNA2-1 promoter in 28 (34.1\%) samples. To assess the occurrence of this hypomethylation in other populations, we obtained an independent genomic DNA methylation dataset (GSE168406) generated from 136 peripheral blood samples of Indian pregnant women who delivered at term $(n=68)$ or preterm $(n=68)$. The methylation status of 6 probes at VTRNA2-1 promoter in this dataset was then analysed (supplementary methods) and methylation levels of cg04481923, cg18678645, cg00124993, and cg26896946 were significantly elevated in PTB compared to $\mathrm{TB}$ (data not shown). In addition, hypomethylation occurred at $25 \% \sim 48.5 \%$ of blood samples (Table S5), on an average of $34.2 \%$ of the population, which was consistent with our statistic of $34.1 \%$ in the Korean population. These results indicate that elevation of VTRNA2-1 promoter is associated with PTB and the hypomethylation of VTRNA2-1 promoter is common in both Korean and Indian populations.

We acknowledge several limitations of our study, including the paucity of clinical and demographic data, for 
Table 6 Clinical characteristics of VTRNA2-1 hypomethylation and elevated methylation groups $(n=82)$

\begin{tabular}{|c|c|c|c|c|c|c|}
\hline & \multicolumn{3}{|c|}{ Hypomethylation $\dagger$} & \multicolumn{3}{|c|}{ Elevated methylation $\neq$} \\
\hline & Term $(n=17)$ & Preterm $(n=11)$ & $p$-value & Term $(n=22)$ & Preterm $(n=32)$ & $p$-value \\
\hline Maternal age & $31.7 \pm 2.7$ & $28.2 \pm 2.5$ & 0.002 & $31.7 \pm 3.1$ & $31.9 \pm 4.7$ & 0.812 \\
\hline BMl & $26.3 \pm 3.3$ & $24.5 \pm 5.4$ & 0.320 & $25.8 \pm 3.3$ & $23.5 \pm 3.4$ & 0.024 \\
\hline Gravidity* & & & 0.954 & & & 0.412 \\
\hline 0 & $6(35.3)$ & $4(36.4)$ & & $10(45.5)$ & $11(34.4)$ & \\
\hline 1 & $11(64.7)$ & $7(63.6)$ & & $12(54.5)$ & $21(65.6)$ & \\
\hline Parity* & & & 0.761 & & & 0.026 \\
\hline Nulliparous & $9(52.9)$ & $5(45.5)$ & & $13(59.1)$ & $17(53.1)$ & \\
\hline Multiparous & $8(47.1)$ & $6(54.5)$ & & $9(40.9)$ & $15(46.9)$ & \\
\hline Delivery season* & & & 0.775 & & & 0.009 \\
\hline Spring, $n(\%)$ & $4(23.5)$ & $4(36.4)$ & & $1(4.5)$ & $8(25.0)$ & \\
\hline Summer, n (\%) & $1(5.9)$ & $0(0.0)$ & & $2(9.1)$ & $11(34.4)$ & \\
\hline Autumn, $n(\%)$ & $9(52.9)$ & $5(45.5)$ & & $10(45.5)$ & $8(25.0)$ & \\
\hline Winter, n (\%) & $3(17.6)$ & $2(18.2)$ & & $9(40.9)$ & $5(15.6)$ & \\
\hline Mode of delivery* & & & 0.435 & & & 0.264 \\
\hline Vaginal, $n$ (\%) & $9(52.9)$ & $8(72.7)$ & & $15(68.2)$ & $16(50.0)$ & \\
\hline C-section, $n(\%)$ & $8(47.1)$ & $3(27.3)$ & & $7(31.8)$ & $16(50.0)$ & \\
\hline Education & & & 0.417 & & & 0.226 \\
\hline Below high school & $3(17.6)$ & $3(37.5)$ & & $5(22.7)$ & $10(47.6)$ & \\
\hline Above college & $14(82.4)$ & $5(62.5)$ & & $17(77.3)$ & $11(52.4)$ & \\
\hline Gestational age & $39.4 \pm 1.0$ & $29.3 \pm 2.4$ & $<0.001$ & $39.4 \pm 1.4$ & $29.1 \pm 2.9$ & $<0.001$ \\
\hline WBC $\left(\times 10^{3}\right)$ & $10.3 \pm 3.3$ & $11.9 \pm 3.0$ & 0.218 & $9.9 \pm 2.9$ & $12.3 \pm 4.0$ & 0.028 \\
\hline Ureaplasma* & & & 0.005 & & & 0.002 \\
\hline Negative & $15(88.2)$ & $4(36.4)$ & & $22(100.0)$ & $18(56.3)$ & \\
\hline Positive & $0(0.0)$ & $5(45.5)$ & & $0(0.0)$ & $8(25.0)$ & \\
\hline \multicolumn{7}{|l|}{ Birth outcome } \\
\hline Body weight (kg) & $3.5 \pm 0.4$ & $1.2 \pm 0.3$ & $<0.001$ & $3.2 \pm 0.3$ & $1.4 \pm 0.5$ & $<0.001$ \\
\hline Sex* & & & 0.934 & & & 0.783 \\
\hline Male, $n(\%)$ & $9(52.9)$ & $6(54.5)$ & & $11(40.0)$ & $18(56.3)$ & \\
\hline Female, $n(\%)$ & $8(47.1)$ & $5(45.5)$ & & $11(60.0)$ & $14(43.8)$ & \\
\hline Apgar 1 min & $9.3 \pm 0.9$ & $6.3 \pm 1.8$ & $<0.001$ & $9.7 \pm 0.5$ & $5.7 \pm 2.5$ & $<0.001$ \\
\hline Apgar 5 min & $9.9 \pm 0.3$ & $7.8 \pm 1.6$ & $<0.001$ & $10.0 \pm 0.0$ & $7.4 \pm 2.2$ & $<0.001$ \\
\hline
\end{tabular}

Data are presented as the mean \pm SD for continuous variables and as $n(\%)$ for categorical variables $B M I$ body mass index

${ }^{\text {a }}$ Student's t-test, $p<0.05$

${ }^{*} x^{2}$ test, $p<0.05$

HHypomethylation, $<13 \%$ methylation of VTRNA2-1

fElevated methylation, methylation level of $30-60 \%$

example on smoking status, alcohol consumption, psychiatric disorders and the use of other drugs or medications. We were only able to demonstrate a correlation between methylation and expression of VTRNA2-1 in a small subset of CpG sites; moreover, the sample size was small due to the limited availability of RNA from maternal blood samples. Finally, when we analysed the VTRN A2-1 methylation level using a simple linear regression model that included the cell composition percentages as covariates, the VTRNA2-1 methylation level was not affected by cell composition $(p>0.05)$. However, we did not analyse DNA methylation status according to cellular heterogeneity, because we did not sort the samples by blood cell type at the time of collection.

\section{Conclusions}

This study suggests that VTRNA2-1 methylation, identified through genome-wide DNA methylation analysis 
and verification by pyrosequencing in blood cells, may be associated with PTB. Our findings suggest that elevated methylation of VTRNA2-1 promoter appear more frequently in pPROM than in PTL, and may be associated with increased risk of PTB. Thus, the evaluation of genes containing these differentially methylated sites may be useful to identify biological pathways involved in PTB, thereby facilitating the identification of clinically informative biomarkers for the prediction of PTB.

\section{Methods}

\section{Study population}

We conducted a case-control study of 10 pregnant women with term $(n=5)$ and preterm deliveries $(n=5)$ at Ewha Womans University Mokdong Hospital (Seoul, Korea) to screen changes in methylation level. Maternal peripheral blood samples from participants were collected at the time of delivery, and the birth outcome was followed (Table S2). DNA methylation was measured using the Illumina Human Methylation 450 BeadChip. To validate the DM levels, 82 blood samples from women with term $(n=39)$ and preterm $(n=43)$ births were examined. All participants gave informed consent, and the study was approved by the Institutional Review Board of Ewha Womans University Mokdong Hospital (Certificate No. EUMC 2014-06-010-003, Samsung Medical Center (SMC 2014-06-094-003), Konkuk University Medical Center (KUH1040034), and Seoul St. Mary's Hospital (KC14TIMI0591). Informed consent was obtained from all subjects involved in the study. Women with multiple births, major birth defects, haemolysis, elevated liver enzymes, a low platelet count (HELLP) syndrome, preeclampsia and gestational diabetes mellitus were excluded. Additionally, pregnant women with inflammatory and metabolic diseases or hormonal problems also were excluded. Gestational age was determined using the first day of the last menstrual period and ultrasound examination.

\section{DNA preparation and genome-wide DNA methylation analysis}

Maternal blood was collected in EDTA tubes, and the plasma was separated and stored at $-80{ }^{\circ} \mathrm{C}$. Genomic DNA was extracted from blood samples using the QIAG EN Mini Kit (QIAGEN, Valencia, CA, USA) following the manufacturer's protocol. The quality of the extracted DNA was evaluated using agarose gel electrophoresis. To analyse DNA methylation, $\sim 700 \mathrm{ng}$ genomic DNA was bisulphite-converted using the Zymo EZ DNA Methylation Kit (Zymo Research, Irvine, CA, USA), amplified, fragmented, and hybridised to the Illumina Infinium HumanMethylation450 BeadChip (Illumina, San Diego, CA, USA) following the manufacturer's protocol. After washing, the BeadChips were scanned with the HiScan SQ System (Illumina). Scanned images were processed to determine the signal intensity and $\beta$ values were calculated using Genome Studio software (Illumina). The $\beta$-value, as defined below, was used to measure methylation levels on a scale from 0 to 1 :

$$
\beta=\frac{\operatorname{Max}(\text { Signal B.0 })}{\operatorname{Max}(\text { Signal A.0) }+ \text { Max }(\text { Signal B.0 })+100}
$$

Max (Signal $A, 0)$ indicates the signal intensity of the unmethylated allele, and Max (Signal B,0) indicates the signal intensity of the methylated allele. A constant bias of 100 was added to regularise the $\beta$-value. The $\beta$-values were calculated; normalisation, filtration, and statistical analyses were performed using GeneSpring ver. 7.3 (Agilent Technologies, Santa Clara, CA, USA). The normalised $\beta$-value of all $\mathrm{CpG}$ sites in the two groups (term vs. preterm) were statistically evaluated using Welch's t-test $(p<0.05)$. We accounted for multiple testing by controlling for the false discovery rate (FDR). The FDR was controlled using the Benjamini-Hochberg correction $(q<0.05)$.

\section{DM analysis by pyrosequencing}

DM levels measured by the genome-wide methylation array were validated in maternal term $(n=39)$ and preterm $(n=43)$ blood by pyrosequencing. The cg04481923 site was amplified using a primer set designed using PSQ Assay Design software (Biotage AB, Uppsala, Sweden) (Table S3). Genomic DNA was bisulphite-converted according to the manufacturer's instructions with an EZ DNA Methylation Kit (ZYMO Research, Irvine, CA, USA). An EpiTect PCR Control DNA Set (Qiagen) was used as a methylated/unmethylated control. The percentage of methylated cells in each region was quantified using the PyroMark ID pyrosequencer (Qiagen) and Pyro Q-CpG Software (Figure S2). The software incorporates controls to check for completed bisulphite conversions, and provides an adequate signal over background noise. All samples were run in duplicate and average values were calculated. The details of the pyrosequencing methodology have previously been reported [46, 47].

\section{RNA isolation and quantitative real-time polymerase chain reaction}

Total RNA from maternal blood $(n=40)$ was extracted using the Easy-BLUE ${ }^{\mathrm{m}}$ Kit (iNtRON Biotechnology, Sungnam, Korea) according to the manufacturer's instructions. RNA was reverse transcribed using $1 \mu \mathrm{g}$ total RNA in a $25 \mu \mathrm{L}$ reaction mixture containing $1 \mu \mathrm{L} 10$ pM oligonucleotide primer, $5 \mu \mathrm{L} 10 \times$ reverse transcription buffer, $5 \mu \mathrm{L} 2.5 \mathrm{mM}$ dNTPs, $1 \mu \mathrm{L} 20 \mathrm{U}$ RNase inhibitor, and $1 \mu \mathrm{L} 200 \mathrm{U}$ Moloney murine leukaemia 
virus reverse transcriptase (M-MLV RT) (Promega, Madison, WI, USA) for $60 \mathrm{~min}$ at $42{ }^{\circ} \mathrm{C}$. Real-time quantitative-polymerase chain reaction (qPCR) was performed using synthesised cDNA as a template, genespecific primers (VTRNA2-1), and Power SYBR Green PCR Master Mix (Applied Biosystems, Foster City, California, USA). The reactions (including the no-template controls) were run in duplicate on the ABI PRISM 7000 sequence detection system (Applied BioSystems) using glyceraldehyde-3-phosphate dehydrogenase (GAPDH) as an internal reference for normalisation of target gene mRNA expression. The PCR conditions were as follows: denaturation at $95{ }^{\circ} \mathrm{C}$ for $30 \mathrm{~s}, 40$ cycles of denaturation at $95{ }^{\circ} \mathrm{C}$ for $15 \mathrm{~s}$, and annealing/extension at $60{ }^{\circ} \mathrm{C}$ for $1 \mathrm{~min}$. We tested primer specificity by RT-PCR and confirmed it using melting (dissociation) curve analysis. Comparative quantification of each target gene was performed based on the cycle threshold $(\mathrm{CT})$, which was normalised against the CT of GAPDH using the $\Delta \Delta C T$ method. Data are presented as the fold change between groups as the mean \pm standard error of the mean (SEM). The primer sets and melting temperature (Tm) for qPCR are described in Table S4.

\section{Statistical analysis}

The basic characteristics of the study groups were compared using Student's $t$-test for continuous variables and the chi-square test for categorical variables. After pyrosequencing, the DNA methylation levels between the two groups were compared using the Mann-Whitney U-test. DNA methylation levels of VTRNA2-1 were analyzed in two separate groups, a group with hypomethylation $(<13 \%)$ and a group with elevated levels of methylation $(30-60 \%)$ by the level of methylation. We used the Spearman coefficient to analyze the correlation between VTRNA2-1 methylation levels and maternal clinical characteristics. To explore the association between VTRNA2-1 methylation level and PTB, multiple logistic regression was conducted, controlling for maternal age, parity, season, and white blood cell (WBC) count. In addition, the clinical characteristics of the VTRNA2-1 hypo- and elevated methylation groups were analysed using Student's $t$-test and the chi-square test. All analyses were two-tailed, and a $p$-value $<0.05$ was considered statistically significant. All statistical analyses were performed using SPSS software ver. 21.0 (IBM, Armonk, NY, USA).

\section{Abbreviations}

VTRNA2-1: Vault RNA 2-1; PTB: Preterm birth; BMI: Body mass index; PKR: Protein kinase-R; CRP: C-reactive protein; GAPDH: Glyceraldehyde-3phosphate dehydrogenase; CT: Cycle threshold; SEM: Standard error of the mean; pPROM: Preterm premature rupture of membrane; PTL: Preterm labor

\section{Supplementary Information}

The online version contains supplementary material available at https://doi. org/10.1186/s12864-021-07865-y.

Additional file 1: Table S1. Differentially methylated sites between term and preterm samples $(n=1,581)$.

Additional file 2: Table S2. The demographics from the pilot cohort $(n=10)$.

Additional file 3: Table S3. Primer set for pyrosequencing designed by the PSQ Assay Design software.

Additional file 4: Table S4. Primers sets for Quantitative real-time PCR.

Additional file 5: Table S5. Frequency of hypomethylation of the VTRN A2-1 promoter in Indian populations $(n=136)$.

Additional file 6: Figure S1. Comparison of seven differential CpG sites on VTRNA2-1 between women with term and preterm births in initial samples $(n=10)$.

Additional file 7: Figure S2. Schematic representation of the human VTRNA2-1 gene. (a) Identified CpG site of VTRNA2-1 (b) PCR products (c) Pyrogram.

\section{Acknowledgements}

We thank research team in School of Biological Sciences, Seoul National University for analysing the GEO data. We are very grateful to the editor and reviewers who critically evaluated the manuscript and provided constructive comments for improvement.

\section{Authors' contributions}

YAY and KYJ designed the study and wrote the manuscript. YAY and KEJ performed the experiments. YAY, KEJ, and KYJ performed the research and analysed the data. HHS, CSJ, and CSK participated in data analysis. All authors interpreted and discussed the data, reviewed and revised the manuscript, and approved the final version of the manuscript.

\section{Funding}

This study was supported by the Ministry of Health \& Welfare of the Republic of Korea (Fund no. HI18C0378) through the Korea Health Industry Development Institute and supported by funding from the National Research Foundation of Korea (NRF-2020R1A2C3011850) and the BK21 FOUR funded by the Ministry of Education and NRF. The paper (Young-Ah You) was supported by RP-Grant 2018 of Ewha Womans University. The funding bodies played no role in the design of the study and collection, analysis, and interpretation of data and in writing the manuscript.

\section{Availability of data and materials}

The datasets supporting the conclusions of this article are included in the article and its Additional files. The datasets generated and/or analysed during the current study are available in the GEO repository, https://www. ncbi.nlm.nih.gov/geo/ (accession numbers: GSE178609).

\section{Declarations}

Ethics approval and consent to participate

All participants provided informed consent and the study was approved by the Institutional Review Board of Ewha Womans University Mokdong Hospital (Certificate No. EUMC 2014-06-010-003, Samsung Medical Center (SMC 2014-06-094-003), Konkuk University Medical Center (KUH1040034), and Seoul St. Mary's Hospital (KC14TIMI0591). Informed consent was obtained from all subjects involved in the study.

Consent for publication

Not applicable.

\section{Competing interests}

The authors declare that they have no competing interests.

\section{Author details}

${ }^{1}$ Department of Obstetrics and Gynecology, Ewha Medical Research Institute, Ewha Womans University Medical School, 07985 Seoul, Korea. ${ }^{2}$ Graduate 
Program in System Health Science and Engineering, Ewha Womans University, 03760 Seoul, Korea. ${ }^{3}$ Department of Obstetrics and Gynecology, Research Institute of Medical Science, Konkuk University School of Medicine, 143-729 Seoul, Korea. ${ }^{4}$ Department of Obstetrics and Gynecology, Samsung Medical Center, Sungkyunkwan University School of Medicine, 135-710 Seoul, Korea. ${ }^{5}$ College of Medicine, The Catholic University of Korea, 505 Banpo-dong, Seocho-gu, 137-040 Seoul, Korea. ${ }^{6}$ Department of Obstetrics and Gynecology, Ewha Womans University Mok Dong Hospital, 158-051 Seoul, South Korea.

\section{Received: 10 March 2020 Accepted: 30 June 2021} Published online: 10 July 2021

\section{References}

1. Lawn JE, Kinney MV, Belizan JM, Mason EM, McDougall L, Larson J, et al. Born too soon: accelerating actions for prevention and care of 15 million newborns born too soon. Reprod Health. 2013;10:6.

2. Birth Statistics in 2019. Statistis Korea, Daejeon. 2019. http://kostat.go.kr/ portal/korea/kor_nw/1/1/index.board. Accepted 26 Aug 2020.

3. Lee KJ, Sohn S, Hong K, Kim J, Kim R, et al. Maternal, infant, and perinatal mortality statistics and trends in Korea between 2009 and 2017. Obstet Gynecol Sci. 2020;63:623-30.

4. Raju TN, Higgins RD, Stark AR, Leveno KJ. Optimizing care and outcome for late-preterm (near-term) infants: a summary of the workshop sponsored by the National Institute of Child Health and Human Development. Pediatrics. 2006;118:1207-14

5. Park S, You YA, Yun H, Choi SJ, Hwang HS, Choi SK, et al. Cervicovaginal fluid cytokines as predictive markers of preterm birth in symptomatic women. Obstet Gynecol Sci. 2020;63:455-63.

6. Beck S, Wojdyla D, Say L, Betran AP, Merialdi M, Requejo JH, et al. The worldwide incidence of preterm birth: A systematic review of maternal mortality and morbidity. Bull World Health Organ. 2010;88:31-8.

7. Yuan W, Basso O, Sorensen HT, Olsen J. Indicators of fetal growth and infectious disease in childhood-A birth cohort with hospitalization as outcome. Eur J Epidemiol. 2001;17:829-34.

8. Bhutta AT, Cleves MA, Casey PH, Cradock MM. Anand KJ. Cognitive and behavioral outcomes of school-aged children who were born preterm: A meta-analysis. JAMA. 2002;288:728-37.

9. Blencowe H, Cousens S, Chou D, Oestergaard M, Say L, Moller AB, et al. Born too soon: The global epidemiology of 15 million preterm births. Reprod Health. 2013;10(Suppl 1):2.

10. Institute of Medicine (US) Committee on Understanding Premature Birth and Assuring Healthy Outcomes. Causes of Preterm Birth. In: Behrman RE, Butler AS, editors. Preterm Birth: Causes, Consequences, and Prevention. Washington (DC): National Academies Press (US); 2007. p. 87-258.

11. Menon R. Spontaneous preterm birth, a clinical dilemma: etiologic, pathophysiologic and genetic heterogeneities and racial disparity. Acta Obstet Gynecol Scand. 2008;87:590-600.

12. Menon R, Conneely KN, Smith A. DNA methylation: An epigenetic risk factor in preterm birth. Reprod Sci. 2012;19:6-13.

13. Rubens CE, Sadovsky Y, Muglia L, Gravett MG, Lackritz E, Gravett C. Prevention of preterm birth: Harnessing science to address the global epidemic. Sci Transl Medicine. 2014;6:262sr5.

14. Knight AK, Smith AK. Epigenetic biomarkers of preterm birth and its risk factors. Genes (Basel). 2016;7:E15.

15. Rogac M, Peterlin B. Epigenetic signature of chronic maternal stress load during pregnancy might be a potential biomarker for spontaneous preterm birth. Balkan J Med Genet. 2018;21:27-33.

16. Parets SE, Conneely KN, Kilaru V, Fortunato SJ, Syed TA, Saade G, et al. DNA Methylation associated with early spontaneous preterm birth and gestational age. Plos one. 2013;8:e67489.

17. Myte R, Sundkvist A, Van Guelpen B, Harlid S. Circulating levels of inflammatory markers and DNA methylation, an analysis of repeated samples from a population based cohort. Epigenetics. 2019;14:649-59.

18. Stroud LR, Papandonatos GD, Rodriguez D, Meaghan M, Salisbury AM, Phipps MG, et al. Maternal smoking during pregnancy and infant stress response: Test of a prenatal programming hypothesis. Psychoneuroendocrinology. 2014;48:29-40.

19. Vidal AC, Benjamin Neelon SE, Liu Y, Tuli AM, Fuemmeler BF, Hoyo C,et al. Maternal stress, preterm birth, and DNA methylation at imprint regulatory sequences in humans. Genet Epigenet. 2014;6:37-44.
20. Smith AK, Conneely KN, Newport DJ, Kilaru V, Schroeder JW, Pennell PB, et al. Prenatal antiepileptic exposure associates with neonatal DNA methylation differences. Epigenetics Off J DNA Methylation Soc. 2012;7: 458-63.

21. Sharp GC, Lawlor DA, Richmond RC, Fraser A, Simpkin A, Suderman M, et al. Maternal pre-pregnancy BMl and gestational weight gain, offspring DNA methylation and later offspring adiposity: Findings from the Avon Longitudinal Study of Parents and Children. Int J Epidemiol. 2015;44:1288-304.

22. Soubry A, Murphy S, Huang Z, Murtha A, Schildkraut J, Jirtle R, et al. The effects of depression and use of antidepressive medicines during pregnancy on the methylation status of the IGF2 imprinted control regions in the offspring. Clin Epigenetics. 2011;3:2.

23. Kim J, Pitlick MM, Christine PJ, Schaefer AR, Saleme C, Comas B, et al. Genome-wide analysis of DNA methylation in human amnion. Sci World J. 2013;2013:678156.

24. Eckmann-Scholz C, Bens J, Kolarova J, Schneppenheim S, Caliebe A, Heidemann S, von Kaisenberg C, et al. DNA-methylation profiling of fetal tissues reveals marked epigenetic differences between chorionic and amniotic samples. Plos one. 2012;7:e39014.

25. Treppendahl MB, Qiu X, Sogaard A, Yang X, Nandrup-Bus C, Hother C, et al. Allelic methylation levels of the noncoding VTRNA2-1 located on chromosome 5q31.1 predict outcome in AML. Blood. 2012;119:206-16.

26. Cao J, Song Y, Bi N, Shen J, Liu W, Fan J, et al. DNA methylation-mediated repression of miR-886-3p predicts poor outcome of human small cell lung cancer. Cancer Res. 2013;73:3326-35.

27. Lee HS, Lee K, Jang HJ, Lee GK, Park JL, Kim SY, et al. Epigenetic silencing of the non-coding RNA nc886 provokes oncogenes during human esophageal tumorigenesis. Oncotarget. 2014;5:3472-81.

28. Hong X, Sherwood B, Ladd-Acosta C, Peng S, Ji H, Hao K, Burd I, Bartell TR, Wang G, Tsai HJ, Liu X, Ji Y, Wahl A, Caruso D, Lee-Parritz A, Zuckerman B, Wang $X$. Genome-wide DNA methylation associations with spontaneous preterm birth in US blacks: findings in maternal and cord blood samples. Epigenetics. 2018;13(2):163-72.

29. Merid SK, Novoloaca A, Sharp GC, Küpers LK, Kho AT, Roy R, et al. Epigenomewide meta-analysis of blood DNA methylation in newborns and children identifies numerous loci related to gestational age. Genome Med. 2020;12:25.

30. York TP, Latendresse SJ, Jackson-Cook C, Lapato DM, Moyer S, Wolen AR, Roberson-Nay R, Do EK, Murphy SK, Hoyo C, Fuemmeler BF, Strauss JF. Replicated umbilical cord blood DNA methylation loci associated with gestational age at birth. Epigenetics. 2020;15:1243-58.

31. Czamara D, Eraslan G, Page CM, Lahti J, Lahti-Pulkkinen M, Hämäläinen E, et al. Integrated analysis of environmental and genetic influences on cord blood DNA methylation in new-borns. Nat Commun. 2019;10:2548.

32. Han Z, Mulla S, Beyene J, Liao G, McDonald SD. Knowledge Synthesis Group. Maternal underweight and the risk of preterm birth and low birth weight: a systematic review and meta-analyses. Int J Epidemiol. 2011:40:65-101.

33. Aliyu MH, Lynch O, Wilson RE, Alio AP, Kristensen S, Marty PJ, et al. Association between tobacco use in pregnancy and placenta-associated syndromes: A population-based study. Arch Gynecol Obstet. 2011;283:729-34.

34. Silver MJ, Kessler NJ, Hennig BJ, Dominguez-Salas P, Laritsky E, Baker MS, et al. Independent genome-wide screens identify the tumor suppressor VTRNA2-1 as a human epiallele responsive to periconceptional environment. Genome Biol. 2015;16:118.

35. Goldenberg RL, Culhane JF. Preterm birth and periodontal disease. N Engl J Med. 2006:355:1925-7

36. You YA, Kwon EJ, Choi SJ, Hwang HS, Choi SK, Lee SM, Kim YJ. Vaginal microbiome profiles of pregnant women in Korea using a 165 metagenomics approach. Am J Reprod Immunol. 2019;82:e13124.

37. Caughey AB, Robinson JN, Norwitz ER. Contemporary diagnosis and management of preterm premature rupture of membranes. Rev Obstet Gynecol. 2008;1:11-22.

38. Ronzoni S, Steckle V, D'Souza R, Murphy KE, Lye S, Shynlova O. Cytokine Changes in Maternal Peripheral Blood Correlate With Time-to-Delivery in Pregnancies Complicated by Premature Prelabor Rupture of the Membranes. Reprod Sci. 2019;26:1266-76.

39. Lee YS. A novel type of non-coding RNA, nc886, implicated in tumor sensing and suppression. Genomics Inform. 2015;13:26-30.

40. Kong L, Hao Q, Wang Y, Zhou P, Zou B, Zhang Y. Regulation of p53 expression and apoptosis by vault RNA2-1-5p in cervical cancer cells. Oncotarget. 2015;6:28371-88. 
41. Ligthart S, Marzi C, Aslibekyan S, Mendelson MM, Conneely KN, Tanaka T, et al. DNA methylation signatures of chronic low-grade inflammation are associated with complex diseases. Genome Biol. 2016;17:255:1-15.

42. Ahsan M, Ek WE, Rask-Andersen M, Karlsson T, Lind-Thomsen A, Enroth S, et al. The relative contribution of DNA methylation and genetic variants on protein biomarkers for human diseases. PLoS Genet. 2017;13:e1007005.

43. Lee Kim, Kunkeaw N, Jeon SH, Lee I, Johnson BH, Kang GY, et al. Precursor miR-886, a novel noncoding RNA repressed in cancer, associates with PKR and modulates its activity. RNA. 2011;17:1076-89.

44. Berger W, Steiner E, Grusch M, Elbling L, Micksche M. Vaults and the major vault protein: novel roles in signal pathway regulation and immunity. Cell Mol Life Sci. 2009;66:43-61.

45. García MA. Meurs EF, Esteban M. The dsRNA protein kinase PKR: virus and cell control. Biochimie. 2007;89:799-811.

46. Yoo JY, Lee S, Lee HA, Park H, Park YJ, Ha EH, et al. Can proopiomelanocortin methylation be used as an early predictor of metabolic syndrome? Diabetes Care. 2014;37:734-9.

47. Kwon EJ, Lee HA, You YA, Park H, Cho SJ, Ha EH, Kim YJ. DNA methylations of MC4R and HNF4alpha are associated with increased triglyceride levels in cord blood of preterm infants. Medicine. 2016;95:e4590.

\section{Publisher's Note}

Springer Nature remains neutral with regard to jurisdictional claims in published maps and institutional affiliations.

Ready to submit your research? Choose BMC and benefit from:

- fast, convenient online submission

- thorough peer review by experienced researchers in your field

- rapid publication on acceptance

- support for research data, including large and complex data types

- gold Open Access which fosters wider collaboration and increased citations

- maximum visibility for your research: over $100 \mathrm{M}$ website views per year

At $\mathrm{BMC}$, research is always in progress.

Learn more biomedcentral.com/submissions 